\title{
Bionic Study of Variable Viscosity on MHD Peristaltic Flow of Pseudoplastic Fluid in an Asymmetric Channel
}

\author{
Ambreen A. Khan ${ }^{1}$, Saima Muhammad ${ }^{1}$, R. Ellahi ${ }^{1,2 *}$, and Q. M. Zaigham Zia ${ }^{3}$ \\ ${ }^{1}$ Department of Mathematics \& Statistics, FBAS, IIUI, Islamabad, Pakistan \\ ${ }^{2}$ Department of Mechanical Engineering, University of California Riverside, USA \\ ${ }^{3}$ Department of Mathematics, COMSATS Institute of Information Technology Chak Shazad Islamabad, Pakistan
}

(Received 7 February 2016, Received in final form 31 March 2016, Accepted 9 May 2016)

\begin{abstract}
In this paper, the peristaltic flow of Psedoplastic fluid with variable viscosity in an asymmetric channel is examined. The bionic effects by means of magnetohydrodynamics (MHD) are taken into account. The assumptions of long wave length and low Reynolds number are taken into account. The basic equations governing the flow are first reduced to a set of ordinary differential equation by using appropriate transformation for variables and then solve by using perturbation method. The effect of physical parameters on the pressure rise, velocity and pressure gradient are illustrated graphically. The trapping phenomenon is analyzed through stream lines. A suitable comparison has also been made as a limiting case of the considered problem.
\end{abstract}

Keywords : Peristaltic flow, Pseudoplastic fluid, variable viscosity, MHD, asymmetric channel, analytical solutions

\section{Introduction}

Peristaltic is a mechanism of fluid transport generally from lower to higher pressure induced by a progressive wave of area of contraction/expansion which propagates along the length of a flexible tube or channel. Peristaltic transport has attracted a number of researchers because of wide applications in physiology and industry such as chyme motion in the gastrointestinal tract, urine transport from the kidney to bladder, vasomotion of small blood vessels, movement of ovum in the female fallopian tube, transport of spermatozoa and swallowing food over the esophagus. These flows are broadly studied for different geometries by using numerous assumptions; few noteworthy studies on the topic can be seen from the list of references [1-10] and several therein.

Moreover, the hydromagnetic channel flow have attracted many investigators due to its numerous applications such as generator geothermal reservoirs, development of magnetic devices for cell separation, magnetic wound or cancer tumor treatment causing magnetic hyperthermia, magnetic filtration, separation and microfluidic devices. A

CThe Korean Magnetics Society. All rights reserved.

*Corresponding author: Tel: +92-51-9258037

Fax:+92-51-9019510, e-mail: rahmatellahi@yahoo.com number of researchers have studied MHD fluid flow under various physical situations, for instance, the effect of magnetic field on blood flow was reported by Sud et al. [11] and they discussed that the influence of moving magnetic field accelerates the speed of blood. Yldrm and Sezer [12] have studied the effects of partial slip on the peristaltic flow of a MHD Newtonian fluid in an asymmetric channel. A list of relevant studies on the topic can be found from the list of references [13-21].

Furthermore, the pump cannot always be designed as a uniform tube for serving the needs such as biomedical instruments. Hakeem et al. [22] discussed the influence of an endoscope and fluid with variable viscosity on peristaltic motion. Hakeem et al. [23] have also examined the peristaltic flow of a fluid with variable viscosity under the effect of magnetic field.

In all of the above mentioned studies fluid viscosity is assumed to be constant whereas in industrial system mostly fluids have variable viscosity behavior [24]. In order to fill this gap, the present work deals with the flow of Pseudoplastic fluid having simultaneous effects of variable viscosity and MHD in an asymmetric channel under the assumption of long wave length and low Reynolds number.

The paper goes in the following style. In Section 2, the formulation of the problem is presented. The resulting 
nonlinear problem is solved by means of regular perturbation method in Sections 3. The effects of essential physical parameters are demonstrated through graphs in Section 4. Finally, Section 5 concludes main finings.

\section{Mathematical Formulation of the Problem}

We consider the Pseudoplastic fluid with variable viscosity in an asymmetric channel having width $d_{1}+d_{2}$. The fluid is electrically conducting in the presence of a constant magnetic field $B_{0}$ applied in the perpendicular direction. Due to small magnetic Reynolds number, the induced magnetic field is neglected. The flow is generated by sinusoidal wave trains propagating with constant speed $c$ along the channel walls. A rectangular coordinate system $(X, Y)$ is chosen such that $X$ - axes lies along the center line of the channel and $Y$ - axis transverse to it. The geometry of the wall surface is defined as

$$
\begin{aligned}
& Y=H_{1}=d_{1}+a_{1} \cos \left[\frac{2 \pi}{\lambda}(\bar{X}-c \bar{t})\right], \text { upper wall } \\
& \bar{Y}=H_{2}=-d_{2}-b_{1} \cos \left[\frac{2 \pi}{\lambda}(\bar{X}-c \bar{t})+\varphi\right], \text { lower wall }
\end{aligned}
$$

in which $c$ is the velocity of the wave, $\lambda$ is the wavelength, $a_{1}$ and $b_{1}$ are the amplitudes of the waves, $\phi$ is the phase difference.

The equations governing the flow are

$$
\begin{aligned}
& \nabla \cdot \overline{\mathbf{V}}=0, \\
& \rho \frac{d \mathbf{V}}{d t}=\operatorname{div} \mathbf{T}+\mathbf{J} \times \mathbf{B},
\end{aligned}
$$

where $\mathbf{J}$ is the current density and $\mathbf{B}$ is the total magnetic field in which $\rho$ is the fluid density, $d / d t$ is the material derivative and $\mathbf{T}$ is the Cauchy stress tensor. The constitutive equations for an incompressible Pseudoplastic fluid are

$$
\overline{\mathbf{T}}=-\bar{P} \overline{\mathbf{I}}+\overline{\mathbf{S}}
$$

where $\bar{P}$ is the pressure, $\overline{\mathbf{I}}$ is the identity tensor and $\overline{\mathbf{S}}$ is the extra stress tensor given by

$$
\begin{aligned}
& \overline{\mathbf{S}}+\bar{\lambda} \overline{\mathbf{S}}^{\nabla}+\frac{1}{2}\left(\bar{\lambda}_{1}-\bar{\mu}_{1}\right)\left(\overline{\mathbf{A}}_{1} \overline{\mathbf{S}}+\overline{\mathbf{S}}_{1}\right)=\mu \overline{\mathbf{A}}_{1}, \\
& \overline{\mathbf{S}}^{\nabla}=\frac{d \overline{\mathbf{S}}}{d t}-\overline{\mathbf{S}} \bar{L}^{\overline{\mathbf{T}}}-\bar{L} \overline{\mathbf{S}}, \\
& \overline{\mathbf{L}}=\operatorname{grad} \overline{\mathbf{V}}, \overline{\mathbf{A}}_{1}=\overline{\mathbf{L}}+\overline{\mathbf{L}}^{\overline{\mathbf{T}}},
\end{aligned}
$$

where $\mu, \overline{\mathbf{S}}^{\nabla}, \overline{\mathbf{A}}_{1}, \bar{\mu}_{1}$ and $\bar{\lambda}_{1}$ are the dynamic viscosity, the upper-convective derivative, the first Rivlin-Ericksen tensor and the relaxation times. We introduce the following transformation between fix and wave frames

$$
\bar{x}=\bar{X}-c \bar{t}, \bar{y}=\bar{Y}, \bar{u}=\bar{U}-c, \bar{v}=\bar{V}, \bar{p}(\bar{x})=\bar{p}(\bar{X}, \bar{t}),
$$

where $\bar{U}, \bar{V}, \bar{P}$ are the velocity components, pressure in the laboratory frame and $\bar{u}, \bar{v}, \bar{p}$ are the velocity components, pressure in the wave frame respectively. The appropriate equations describing the flow in the wave frame are

$$
\begin{aligned}
& \frac{\partial \bar{u}}{\partial \bar{x}}+\frac{\partial \bar{v}}{\partial \bar{y}}=0 \text {, } \\
& \rho\left[\bar{u} \frac{\partial}{\partial \bar{x}}+\bar{v} \frac{\partial}{\partial \bar{y}}\right] \bar{u}=-\frac{\partial \bar{p}}{\partial \bar{x}}+\frac{\partial \bar{S}_{x x}}{\partial \bar{x}}+\frac{\partial \bar{S}_{x y}}{\partial \bar{y}}-\sigma B_{0}^{2}(\bar{u}+c) \\
& \rho\left[\bar{u} \frac{\partial}{\partial \bar{x}}+\bar{v} \frac{\partial}{\partial \bar{y}}\right] \bar{v}=-\frac{\partial \bar{p}}{\partial \bar{y}}+\frac{\partial \bar{S}_{y x}}{\partial \bar{x}}+\frac{\partial \bar{S}_{y y}}{\partial \bar{y}}-\sigma B_{0}^{2} v, \\
& 2 \bar{\mu}(y) \frac{\partial \bar{u}}{\partial \bar{x}}=\bar{S}_{\overline{x x}}+\bar{\lambda}_{1}\left(\bar{u} \frac{\partial \bar{S}_{\overline{x x}}}{\partial \bar{x}}+\bar{v} \frac{\partial \bar{S}_{\overline{x x}}}{\partial \bar{y}}-2 \frac{\partial \bar{u}}{\partial \bar{x}} \bar{S}_{\overline{x x}}-2 \frac{\partial \bar{u}}{\partial \bar{y}} \bar{S}_{\overline{x y}}\right) \\
& \frac{1}{2}\left(\bar{\lambda}_{1}-\bar{\mu}_{1}\right)\left(4 \bar{S}_{\overline{x x}} \frac{\partial \bar{u}}{\partial \bar{x}}+2 \bar{S}_{\overline{x y}}\left(\frac{\partial \bar{u}}{\partial \bar{y}}+\frac{\partial \bar{v}}{\partial \bar{x}}\right)\right), \\
& 2 \bar{\mu}(y) \frac{\partial \bar{v}}{\partial \bar{y}}=\bar{S}_{\overline{y y}}+\bar{\lambda}_{1}\left(\bar{u} \frac{\partial \bar{S}_{\overline{y y}}}{\partial \bar{x}}+\bar{v} \frac{\partial \bar{S}_{\overline{y y}}}{\partial \bar{y}}-2 \frac{\partial \bar{v}}{\partial \bar{y}} \bar{S}_{\overline{y y}}-2 \frac{\partial \bar{v}}{\partial \bar{x}} \bar{S}_{\overline{x y}}\right) \\
& +\frac{1}{2}\left(\bar{\lambda}_{1}-\bar{\mu}_{1}\right)\left(4 \bar{S}_{\overline{y y}} \frac{\partial \bar{v}}{\partial \bar{y}}+2 \bar{S}_{\overline{x y}}\left(\frac{\partial \bar{u}}{\partial \bar{y}}+\frac{\partial \bar{v}}{\partial \bar{x}}\right)\right), \\
& \bar{\mu}(y)\left(\frac{\partial \bar{u}}{\partial \bar{y}}+\frac{\partial \bar{v}}{\partial \bar{x}}\right)=\bar{S}_{\overline{x y}}+\bar{\lambda}_{1}\left(\bar{u} \frac{\partial \bar{S}_{\overline{x y}}}{\partial \bar{x}}+\bar{v} \frac{\partial \bar{S}_{\overline{x y}}}{\partial \bar{y}}+\frac{\partial \bar{v}}{\partial \bar{x}} \bar{S}_{\overline{x x}}-\frac{\partial \bar{u}}{\partial \bar{y}} \bar{S}_{\overline{y y}}\right) \\
& +\frac{1}{2}\left(\bar{\lambda}_{1}-\bar{\mu}_{1}\right)\left(\left(\bar{S}_{\overline{x x}}+\bar{S}_{\overline{y y}}\right)\left(\frac{\partial \bar{u}}{\partial \bar{y}}+\frac{\partial \bar{v}}{\partial \bar{x}}\right)\right) .
\end{aligned}
$$

Making use of following dimensionless quantities:

$$
\begin{aligned}
& x=\frac{\bar{x}}{\lambda}, h_{1}=\frac{H_{1}}{d_{1}}, y=\frac{\bar{y}}{d_{1}}, h_{2}=\frac{H_{2}}{d_{1}}, p=\frac{d_{1}^{2} \bar{p}}{\mu c \lambda}, \\
& u=\frac{\bar{u}}{c}, \delta=\frac{d_{1}}{\lambda}, \operatorname{Re}=\frac{\rho c d_{1}}{\mu}, d=\frac{d_{2}}{d_{1}}, \mu(y)=\frac{\bar{\mu}(y)}{\mu_{0}}, \\
& a=\frac{a_{1}}{d_{1}}, b=\frac{b_{1}}{d_{1}}, S=\frac{\bar{S} d_{1}}{\mu c}, K=\frac{k}{d_{1}^{2}}, \sigma=\frac{1}{\sqrt{K}}, \lambda_{1}=\frac{\bar{\lambda}_{1} c}{d_{1}}, \\
& \sigma=\frac{1}{\sqrt{K}}, \mu_{1}=\frac{\bar{\mu}_{1} c}{d_{1}}, M=\frac{\sigma B_{0}^{2} d_{1}^{2}}{\mu_{0}}, u=\frac{\partial \psi}{\partial y}, v=-\delta \frac{\partial \psi}{\partial x} .
\end{aligned}
$$

Under the lubrication approach, the incompressibility condition is automatically satisfied and Eqs. (11)-(15) reduced to

$$
\frac{\partial p}{\partial x}=\frac{S_{x y}}{\partial y}-M^{2}\left(\frac{\partial \psi}{\partial y}+1\right),
$$




$$
\begin{aligned}
& \frac{\partial p}{\partial y}=0, \\
& S_{x x}=\left(\lambda_{1}+\mu_{1}\right) \frac{\partial^{2} \psi}{\partial y^{2}} S_{x y}, S_{y y}=\left(-\lambda_{1}+\mu_{1}\right) \frac{\partial^{2} \psi}{\partial y^{2}} S_{x y}, \\
& S_{x y}=\frac{\mu(y) \frac{\partial^{2} \psi}{\partial y^{2}}}{1+\xi\left(\frac{\partial^{2} \psi}{\partial y^{2}}\right)^{2}} .
\end{aligned}
$$

The effect of variable viscosity on the peristaltic flow can be investigated for $\mu(y)$.

For the present investigation, we assume the viscosity effects in the dimensionless form as

$$
\mu(y)=e^{-\alpha y}=1-\alpha y, \quad \alpha<<1,
$$

where $\alpha$ is the viscosity parameter. Using Eq. (20) into Eq. (17), we can write

$$
\frac{\partial p}{\partial x}=\frac{\partial}{\partial y}\left[(1-\alpha y) \frac{\partial^{2} \psi}{\partial y^{2}}\left\{1-\xi\left(\frac{\partial^{2} \psi}{\partial y^{2}}\right)^{2}\right\}\right]-M^{2}\left(\frac{\partial \psi}{\partial y}+1\right) .
$$

By eliminating pressure $p$ from Eq. (22) reduces to

$$
\frac{\partial^{2}}{\partial y^{2}}\left[(1-\alpha y) \frac{\partial^{2} \psi}{\partial y^{2}}\left\{1-\xi\left(\frac{\partial^{2} \psi}{\partial y^{2}}\right)^{2}\right\}\right]-M^{2} \frac{\partial^{2} \psi}{\partial y^{2}}=0 .
$$

The boundary conditions in the wave frame are then written as

$$
\begin{aligned}
& \psi=\frac{F}{2}, \frac{\partial \psi}{\partial y}=-1 \text { at } y=h_{1}=1+a \cos (2 \pi x), \\
& \psi=-\frac{F}{2}, \frac{\partial \psi}{\partial y}=-1 \text { at } y=h_{2}=-d-b \cos (2 \pi x+\varphi) .
\end{aligned}
$$

The mean flow rate in wave frame of reference is given by

$$
F=\int_{h_{2}}^{h_{1}} \frac{\partial \psi}{\partial y} d y
$$

which is related with dimensionless mean flow rate in the laboratory frame $\theta$, by the relation

$$
\theta=F+1+d,
$$

and the dimensionless pressure rise $\Delta p$ is defined by

$$
\Delta p=\int_{0}^{1} \frac{d p}{d x} d x
$$

\section{Solution of the Problem}

For analytical solutions, we expand $\psi, F, d p / d x$ in terms of small parameters $\xi$ and $\alpha$ as

$$
\begin{aligned}
\psi & =\psi_{0}+\xi \psi_{1}+O\left(\xi^{2}\right), \\
F & =F_{0}+\xi F_{1}+O\left(\xi^{2}\right), \\
\frac{d p}{d x} & =\frac{d p_{0}}{d x}+\xi \frac{d p_{1}}{d x}+O\left(\xi^{2}\right),
\end{aligned}
$$

where

$$
\begin{aligned}
\psi_{0} & =\psi_{00}+\alpha \psi_{01}+O\left(\alpha^{2}\right), \\
\psi_{1} & =\psi_{10}+\alpha \psi_{11}+O\left(\alpha^{2}\right), \\
\frac{d p_{0}}{d x} & =\frac{d p_{00}}{d x}+\alpha \frac{d p_{01}}{d x}+O\left(\alpha^{2}\right), \\
\frac{d p_{1}}{d x} & =\frac{d p_{10}}{d x}+\alpha \frac{d p_{11}}{d x}+O\left(\alpha^{2}\right), \\
F_{0} & =F_{00}+\alpha F_{01}+O\left(\alpha^{2}\right), \\
F_{1} & =F_{10}+\alpha F_{11}+O\left(\alpha^{2}\right) .
\end{aligned}
$$

Using Eqs. (29) and (30) into Eqs. (22) and (23 and equating the coefficients of like terms of $\xi$ and $\alpha$, we have the following system of equations.

\subsection{Zeroth order system}

$$
\begin{aligned}
& \frac{\partial p_{00}}{\partial x}=\frac{\partial^{3} \psi_{00}}{\partial y^{3}}-M^{2} \frac{\partial \psi_{00}}{\partial y}, \\
& \frac{\partial^{4} \psi_{00}}{\partial y^{4}}-M^{2} \frac{\partial^{2} \psi_{00}}{\partial y^{2}}=0 .
\end{aligned}
$$

The boundary conditions can also be obtained by substituting Eqs. (29) and (30) into Eqs. (24) and (25). Thus, by expanding Taylor series about the mean position, one can obtain the following boundary conditions associated with the zeroth order deformation problems are given as follows:

$$
\begin{aligned}
& \psi_{00}=\frac{F_{00}}{2}, \frac{\partial \psi_{00}}{\partial y}=-1 \text { at } y=h_{1}, \\
& \psi_{00}=-\frac{F_{00}}{2}, \frac{\partial \psi_{00}}{\partial y}=-1 \text { at } y=h_{2} .
\end{aligned}
$$

Solving the Eqs. (31)-(32) with the use of boundary conditions (33) and (34), we obtained the zeroth-order solutions in term of stream function, axial velocity and pressure gradient as

$$
\begin{aligned}
& \psi_{00}=C_{1}+C_{2} y+C_{3} \cosh M y+C_{4} \sinh M y . \\
& u_{00}=C_{2}+M\left(C_{3} \sinh M y+C_{4} \cosh M y\right), \\
& \frac{d p_{0}}{d x}=-M^{2}\left(C_{2}+1\right) .
\end{aligned}
$$

\subsection{First order system}

The governing equations and the associated boundary 
conditions for the first-order are

$$
\begin{aligned}
& \frac{\partial^{4} \psi_{01}}{\partial y^{4}}-M^{2} \frac{\partial^{2} \psi_{01}}{\partial y^{2}}=\frac{\partial^{2}}{\partial y^{2}}\left(y \frac{\partial^{2} \psi_{00}}{\partial y^{2}}\right), \\
& \frac{\partial p_{01}}{\partial x}=\frac{\partial^{3} \psi_{01}}{\partial y^{3}}-M^{2} \frac{\partial \psi_{01}}{\partial y}-\frac{\partial}{\partial y}\left(y \frac{\partial^{2} \psi_{00}}{\partial y^{2}}\right), \\
& \psi_{01}=\frac{F_{01}}{2}, \frac{\partial \psi_{01}}{\partial y}=0 \text { at } y=h_{1}, \\
& \psi_{01}=-\frac{F_{01}}{2}, \frac{\partial \psi_{01}}{\partial y}=0 \text { at } y=h_{2} .
\end{aligned}
$$

Solving the Eqs. (38) and (39) with the use of boundary conditions (40) and (41), the first order solutions for stream function, axial velocity and pressure gradient can be obtained as

$$
\begin{aligned}
\psi_{01}= & C_{5}+C_{6} y+C_{7} \cosh M y+C_{8} \sinh M y \\
& -\frac{y}{4}\left(C_{3} \sinh M y+C_{4} \cosh M y\right) \\
& +\frac{M y^{2}}{4}\left(C_{3} \sinh M y+C_{4} \cosh M y\right), \\
u_{01}= & C_{6}+M\left(C_{7} \sinh M y+C_{8} \cosh M y\right) \\
& -\frac{1}{4}\left(C_{3} \cosh M y+C_{4} \sinh M y\right) \\
& +\frac{1}{4} M y\left(C_{3} \sinh M y+C_{4} \cosh M y\right) \\
& +\frac{M^{2} y^{2}}{4}\left(C_{3} \cosh M y+C_{4} \sinh M y\right), \\
\frac{d p_{01}}{d x}= & -M^{2} C_{6} .
\end{aligned}
$$

\subsection{Second order system}

The governing equations of second order are

$$
\begin{aligned}
& \frac{\partial^{4} \psi_{10}}{\partial y^{4}}-M^{2} \frac{\partial^{2} \psi_{10}}{\partial y^{2}}=\frac{\partial^{2}}{\partial y^{2}}\left(\frac{\partial^{2} \psi_{00}}{\partial y^{2}}\right)^{3}, \\
& \frac{\partial p_{10}}{\partial x}=\frac{\partial^{3} \psi_{10}}{\partial y^{3}}-M^{2} \frac{\partial \psi_{10}}{\partial y}-\frac{\partial}{\partial y}\left(\frac{\partial^{2} \psi_{00}}{\partial y^{2}}\right)^{3} .
\end{aligned}
$$

The corresponding boundary conditions are

$$
\begin{aligned}
& \psi_{10}=\frac{F_{10}}{2}, \frac{\partial \psi_{10}}{\partial y}=0 \text { at } y=h_{1}, \\
& \psi_{10}=-\frac{F_{10}}{2}, \frac{\partial \psi_{10}}{\partial y}=0 \text { at } y=h_{2} .
\end{aligned}
$$

Solving the Eqs. (45) and (46) with the help of boundary conditions given in Eqs. (47) and (48), we get

$$
\begin{aligned}
\psi_{10}= & C_{9}+C_{10} y+C_{11} \cosh M y+C_{12} \sinh M y \\
& +\frac{\sigma^{4}}{8}\left(C_{13} \cosh 3 M y+C_{14} \sinh 3 M y\right) \\
& +3 M^{5} y\left(C_{15} \cosh M y+C_{16} \sinh M y\right), \\
u_{10}= & C_{10}+M\left(C_{11} \sinh M y+C_{12} \cosh M y\right) \\
+ & \frac{3 M^{5}}{8}\left(C_{13} \sinh 3 M y+C_{14} \cosh 3 M y\right), \\
\frac{d p_{10}}{d x}= & -M^{2} C_{10} .
\end{aligned}
$$

where

$$
\begin{aligned}
& L_{1}=\sinh M h_{1}-\sinh M h_{2}, L_{2}=\cosh M h_{1}-\cosh M h_{2}, \\
& L_{3}=L_{2}-M\left(h_{1}-h_{2}\right) \sinh M h_{1}, L_{4}=L_{1}-M\left(h_{1}-h_{2}\right) \cosh M h_{1} \text {, } \\
& C_{1}=\frac{F_{00}}{2}-C_{2} h_{1}-C_{3} \cosh M h_{1}-C_{4} \sinh M h_{1}, C_{7}=-\frac{M L_{8} L_{2}}{M L_{1}}-\frac{C_{30}}{M L_{1}}, \\
& C_{2}=\frac{F_{00}-C_{3} L_{2}-C_{4} L_{1}}{h_{1}-h_{2}}, C_{3}=\frac{-C_{4} L_{2}}{L_{1}}, C_{4}=\frac{L_{1}\left(F_{00+}\left(h_{1}-h_{2}\right)\right)}{L_{1} L_{4}-L_{2} L_{3}}, \\
& C_{5}=\frac{F_{01}}{2}+\frac{C_{12}}{L_{1}}\left(M h_{1} \sinh M\left(h_{1}-h_{2}\right)+1-\cosh M\left(h_{1}-h_{2}\right)\right) \\
& +\frac{C_{30}}{M L_{1}}\left(\cosh M h_{1}-M h_{1} \sinh M h_{2}\right)+h_{1} C_{29}-C_{28}, \\
& C_{6}=\frac{M h_{8} \sinh \left(h_{1}-h_{2}\right)}{L_{1}}+\frac{C_{30} \sinh M h_{1}}{L_{1}}-C_{29}, \\
& C_{8}=\frac{L_{1}}{\left(2 \cosh M\left(h_{1}-h_{2}\right)-2-M\left(h_{1}-h_{2}\right) \sinh M\left(h_{1}-h_{2}\right)\right)} \\
& \left(F_{01}+C_{29}\left(h_{1}-h_{2}\right)-C_{31}+\frac{C_{30}\left(L_{2}-M\left(h_{1}-h_{2}\right) \sinh M h_{1}\right)}{M L_{1}}\right), \\
& C_{9}=\frac{F_{10}}{2}-C_{10} h_{1}-C_{11} \cosh M h_{1}-C_{12} \sinh M h_{1}-C_{17}-C_{18} h_{1}, \\
& C_{10}=\frac{F_{10}-C_{11} L_{2}-C_{12} L_{1}-C_{25}}{h_{1}-h_{2}}, C_{11}=\frac{-C_{26}-C_{12} L_{2}}{L_{1}}, \\
& C_{12}=\frac{F_{10} L_{1}+C_{26} L_{3}-C_{27} L_{1}}{L_{1} L_{4}-L_{2} L_{3}}, \\
& C_{13}=\left(\frac{C_{3}}{2}\right)^{2}+3\left(\frac{C_{4}}{2}\right)^{2}, C_{14}=\left(\frac{C_{4}}{2}\right)^{2}+3\left(\frac{C_{3}}{2}\right)^{2}, \\
& C_{15}=\left(\frac{C_{3}}{2}\right)^{2}\left(\frac{C_{4}}{2}\right)-\left(\frac{C_{4}}{2}\right)^{3}, C_{16}=\left(\frac{C_{3}}{2}\right)^{3}-\left(\frac{C_{3}}{2}\right)\left(\frac{C_{4}}{2}\right)^{2} \text {, } \\
& C_{17}=\frac{M^{4}}{8}\left(C_{13} \cosh 3 M h_{1}+C_{13} \sinh 3 M h_{1}\right), \\
& C_{18}=3 M^{5}\left(C_{15} \cosh 3 M h_{1}+C_{16} \sinh 3 M h_{1}\right) \text {, } \\
& C_{19}=\frac{M^{4}}{8}\left(C_{13} \cosh 3 M h_{2}+C_{14} \sinh 3 M h_{2}\right) \text {, } \\
& C_{20}=3 M^{5}\left(C_{15} \cosh 3 M h_{2}+C_{16} \sinh 3 M h_{2}\right), \\
& C_{21}=\frac{M^{4}}{8}\left(C_{13} \cosh 3 M h_{1}+C_{14} \sinh 3 M h_{1}\right) \text {, }
\end{aligned}
$$




$$
\begin{aligned}
C_{22}= & 3 M^{5}\left(C_{15} \sinh M h_{1}+C_{16} \cosh M h_{1}\right), \\
C_{23}= & \frac{M^{4}}{8}\left(C_{13} \sinh 3 M h_{2}+C_{14} \cosh 3 M h_{2}\right), \\
C_{24}= & 3 M^{5}\left(C_{15} \sinh M h_{2}+C_{16} \cosh M h_{2}\right), \\
C_{25}= & C_{17}-C_{19}+C_{18} h_{1}-C_{20} h_{2}, C_{26}=C_{25}-3 M C_{21}, \\
C_{27}= & 3\left(C_{21}-C_{23}\right)+\frac{1}{M}\left(C_{18}-C_{20}\right)+C_{22} h_{1}-C_{24} h_{2}, \\
C_{31}= & -\frac{C_{3}}{4}\left(h_{1} \cosh M h_{1}-h_{2} \cosh M h_{2}\right)-\frac{C_{4}}{4}\left(h_{1} \sinh M h_{1}-h_{2} \sinh M h_{2}\right) \\
& +\frac{C_{3} M}{4}\left(h_{1}^{2} \sinh M h_{1}-h_{2}^{2} \sinh M h_{2}\right) \\
& +\frac{C_{4} M}{4}\left(h_{1}^{2} \cosh M h_{1}-h_{2}^{2} \cosh M h_{2}\right), \\
C_{29}= & \frac{M h_{1}}{4}\left(C_{3} \sinh M h_{1}-C_{4} \cosh M h_{1}\right)-\frac{1}{4}\left(C_{3} \cosh M h_{1}-C_{4} \sinh M h_{1}\right) \\
& +\frac{M^{2} h_{1}^{2}}{4}\left(C_{3} \cosh M h_{1}-C_{4} \sinh M h_{1}\right), \\
& +\frac{C_{4} M^{2}}{4}\left(h_{1}^{2} \sinh M h_{1}-h_{2}^{2} \sinh M h_{2}\right) . \\
C_{28}= & \frac{C_{1}}{4}\left(C_{3} \cosh M h_{1}+C_{4} \sinh M h_{1}\right)+\frac{M h_{1}^{2}}{4}\left(C_{3} \sinh M h_{1}+C_{4} \cosh M h_{1}\right), \\
C_{30}= & \frac{C_{3} M}{4}\left(h_{1} \sinh M h_{1}-h_{2} \sinh M h_{2}\right)+\frac{C_{3} M}{4}\left(h_{1} \cosh M h_{1}-h_{2} \cosh M h_{2}\right) \\
& -\frac{C_{3}}{4}\left(\cosh M h_{1}-\cosh M h_{2}\right)-\frac{C_{4}}{4}\left(\sinh M h_{1}-\sinh M h_{2}\right) \\
& \left.\cosh M h_{1}-h_{2}^{2} \cosh M h_{2}\right) \\
&
\end{aligned}
$$

\section{Results and Discussion}

The Figs. 1 to 15 have been displayed in order to analyze the effects of pertinent parameter, namely Hartmann number $M$, perturbation parameter $\xi$, viscosity parameter $\alpha$, volume flow rate $\theta$ on velocity profile, pressure rise, pressure gradient and trapping phenomenon. Figure 1 shows the variation of velocity profile for different values of Hartmann number $M$. It is depicted that the velocity decreases by increasing the value of $M$. It is in accordance with the physical expectation that the effect of magnetic field is to decrease the magnitude of the velocity profile because in the presence of magnetic field introduces a so called force the Lorentz force that always acts opposite direction of the flow direction and as a result this type of resisting force slows down the velocity of the fluid. Figures 2 to 4 are prepared to examine the behaviour of perturbation parameter $\xi$, viscosity parameter $\alpha$ and volume flow rate $\theta$ on the velocity profile. Figures 2 depicts that an increase in $\xi$ leads to a decrease in the velocity $u$ at the boundaries of the channel. However at the center of the channel $u$ it increases by increasing the values of $\xi$.

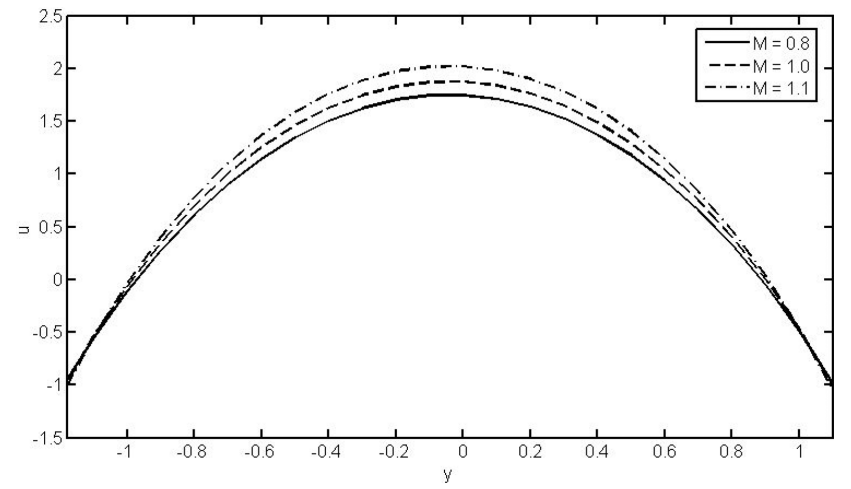

Fig. 1. The variation of $M$ on velocity profile when $\theta=1.4$, $\phi=0.7, \xi=0.2, \alpha=0.01, a=0.6, b=0.9, d=1$.

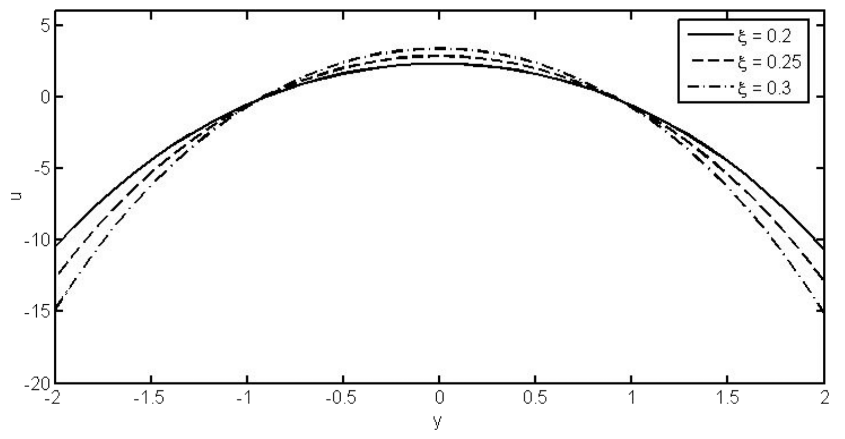

Fig. 2. The variation of $\xi$ on velocity profile when $\theta=1.4$, $\phi=0.7, \xi=0.5, \alpha=0.01, a=0.6, b=0.8, d=1$.

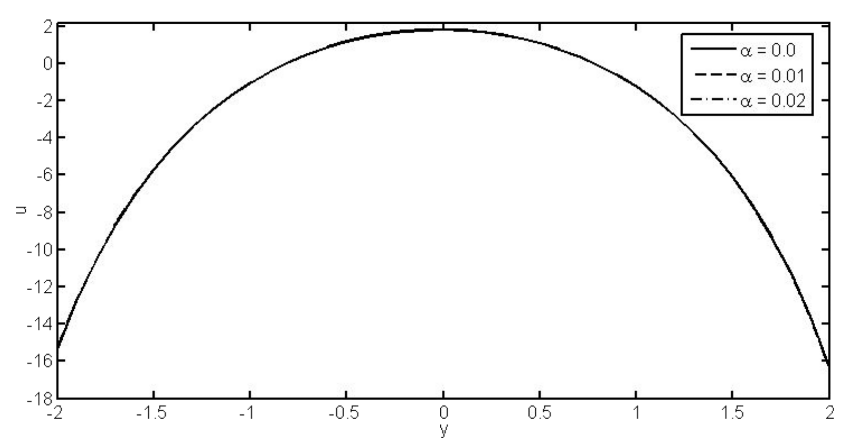

Fig. 3. The variation of $\alpha$ on velocity profile when $\theta=1.4$, $\phi=1, M=0.5, \xi=0.2, a=0.6, b=0.8, d=1$.

Figure 3 illustrates that there is no change in $u$ for different values of $\alpha$. Figure 4 witness that there is a decrease in $u$ at the boundaries of the channel when $\theta$ is increased. At the center of the channel, an increase in $u$ is noticed with the increasing values of $\theta$. An increase can be related with the decrease in the kinematic viscosity. Figures 5 to 7 are plotted to see the effects of $M, \xi$ and $\alpha$ versus pressure rise. These figures illustrate that peristaltic pumping rate increases by increase in $M, \xi$ and $\alpha$. Figure 8 reveals the influence of $\phi$ on pressure rise. This figure predicts that pumping rate decreases with an increase of 


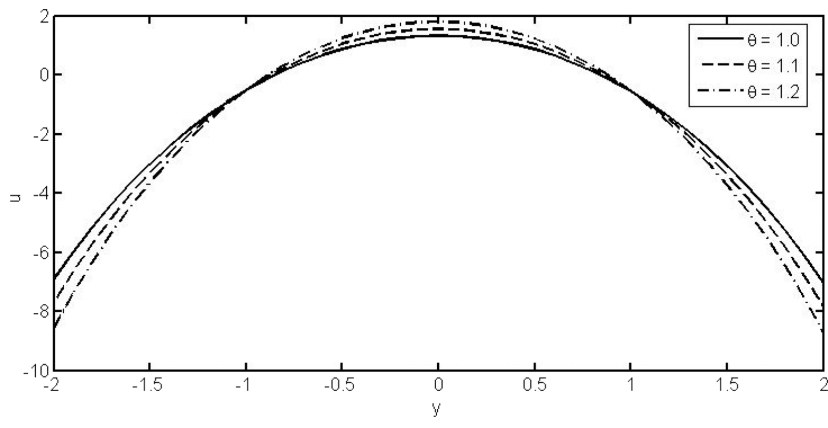

Fig. 4. The variation of $\theta$ on velocity profile when $\phi=0.7$, $M=0.5, \xi=0.2, \alpha=0.01, a=0.6, b=0.8, d=1$.

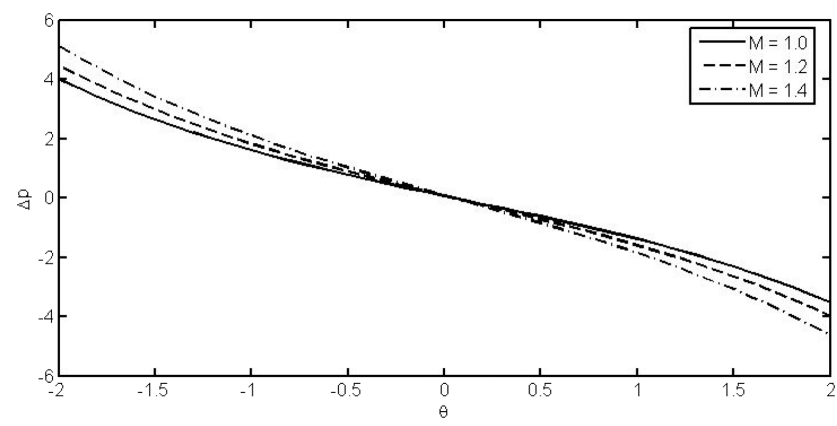

Fig. 5. The variation of $M$ for pressure rise when $\theta$ for $\phi=5$, $\xi=0.02, \alpha=0.3, a=0.2, b=0.2, d=1$.

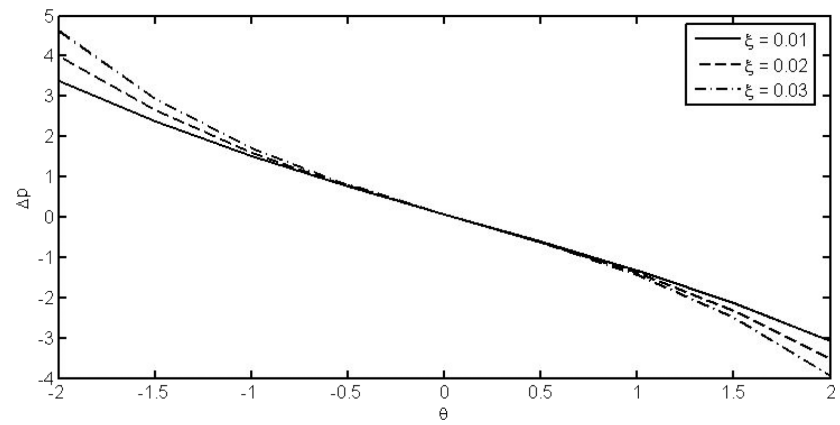

Fig. 6. The variation of $\xi$ for pressure rise when $\theta$ for $\phi=5$, $M=1, \alpha=0.3, a=0.2, b=0.2, d=1$.

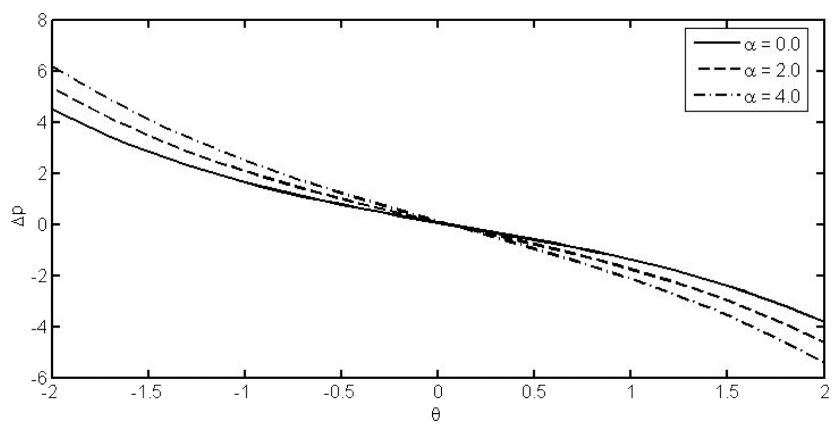

Fig. 7. The variation of $\alpha$ for pressure rise when $\theta$ for $\phi=5$, $M=1, \alpha=0.03, a=0.2, b=0.2, d=1$.

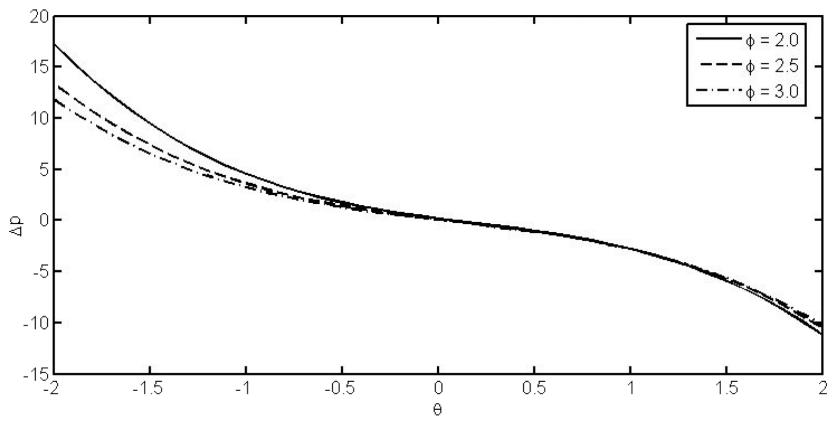

Fig. 8. The variation of $\phi$ for pressure rise when $\theta$ for $M=1$, $\xi=0.03, \alpha=0.3, a=0.2, b=0.4, d=1$.

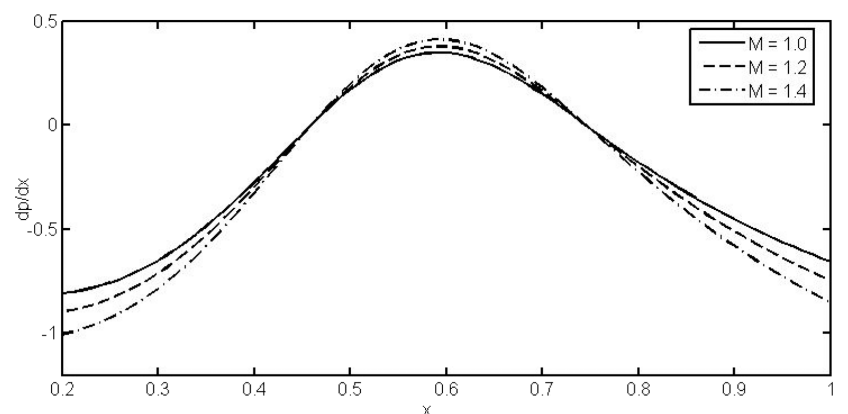

Fig. 9. The variation of $M$ for pressure gradient when $\phi=1$, $\theta=0.3, \xi=0.2, \alpha=0.1, a=0.4, b=0.4, d=1$.

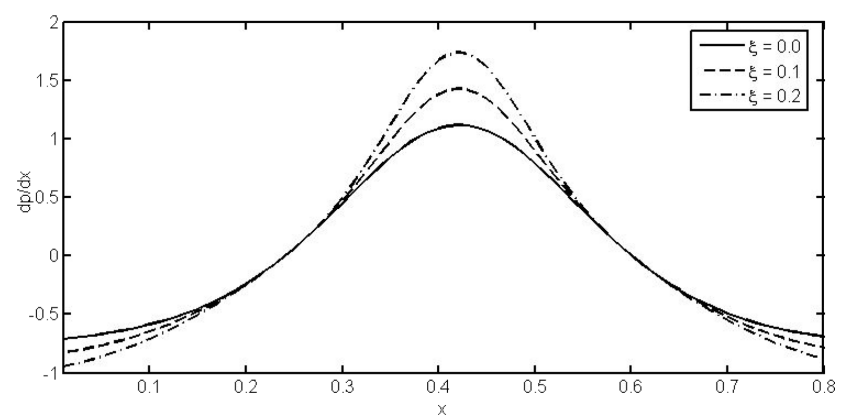

Fig. 10. The variation of $\xi$ for pressure gradient when $\phi=1$, $\theta=0.3, M=1, \alpha=0.1, a=0.4, b=0.4, d=1$.

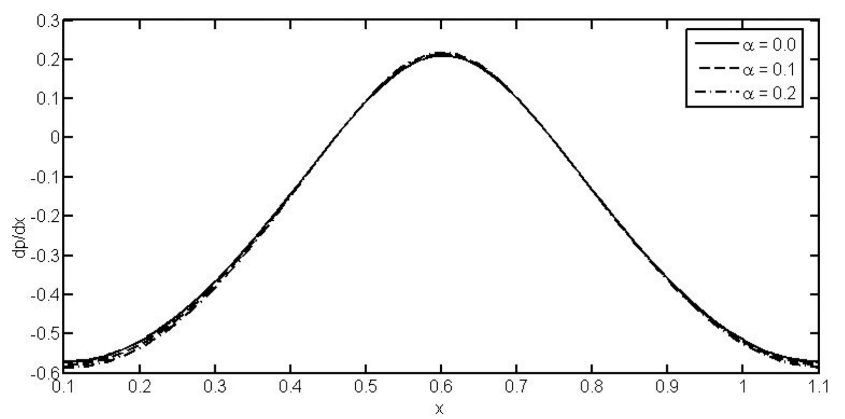

Fig. 11. The variation of $\alpha$ for pressure gradient when $\phi=5$, $\theta=0.2, M=1, \xi=0.2, \alpha=0.4, a=0.2, b=0.2, d=1$. 


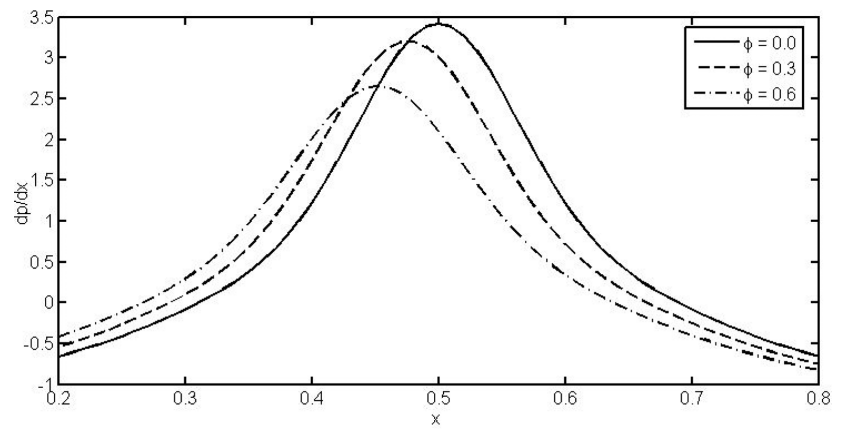

Fig. 12. The variation of $\phi$ for pressure gradient when $\theta=0.3$, $M=1, \xi=0.2, \alpha=0.1, a=0.4, b=0.4, d=1$. $\phi$. All said figures have opposite behavior in co-pumping region. As it is exposed previous that the Lorentz force is acting directly to dump the mainstream velocity, therefore decrease in velocity component in flow direction cusses noticeable decrease of pressure rise in co-pumping region. Figures 9 to 11 represent the behaviour of pressure gradient for different values of $M, \xi$ and $\alpha$. The effects of $M, \xi$ and $\alpha$ on the pressure gradient is plotted in Figures 9 to 11 . It is noticed that the absolute value of pressure gradient increases with an increase in $M, \xi$ and $\alpha$ whereas near the channel walls pressure gradient is small. It is due to the fact that flow can easily pass in the middle of the

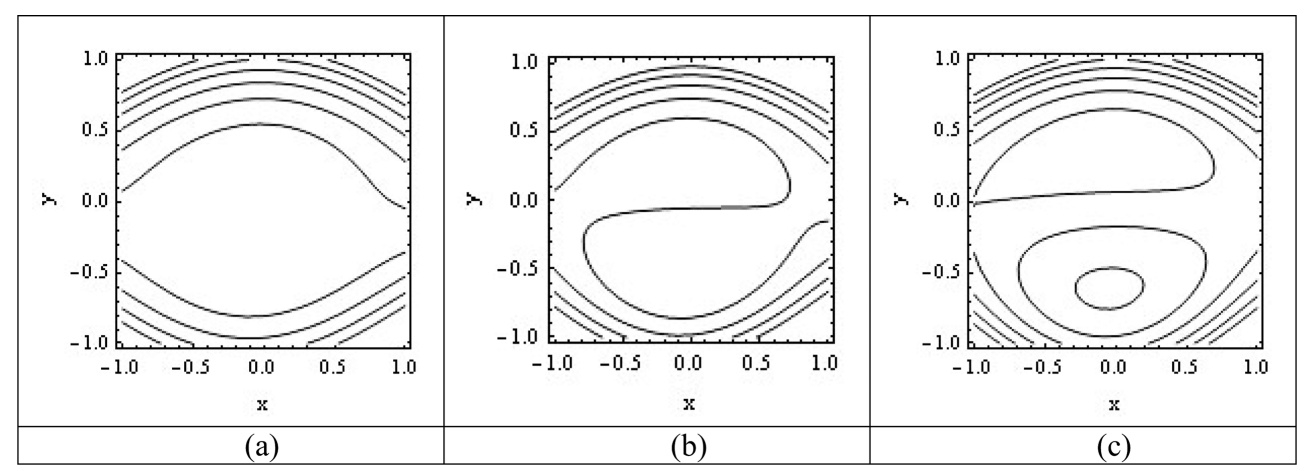

Fig. 13. Streamlines for viscosity parameter $\alpha$.

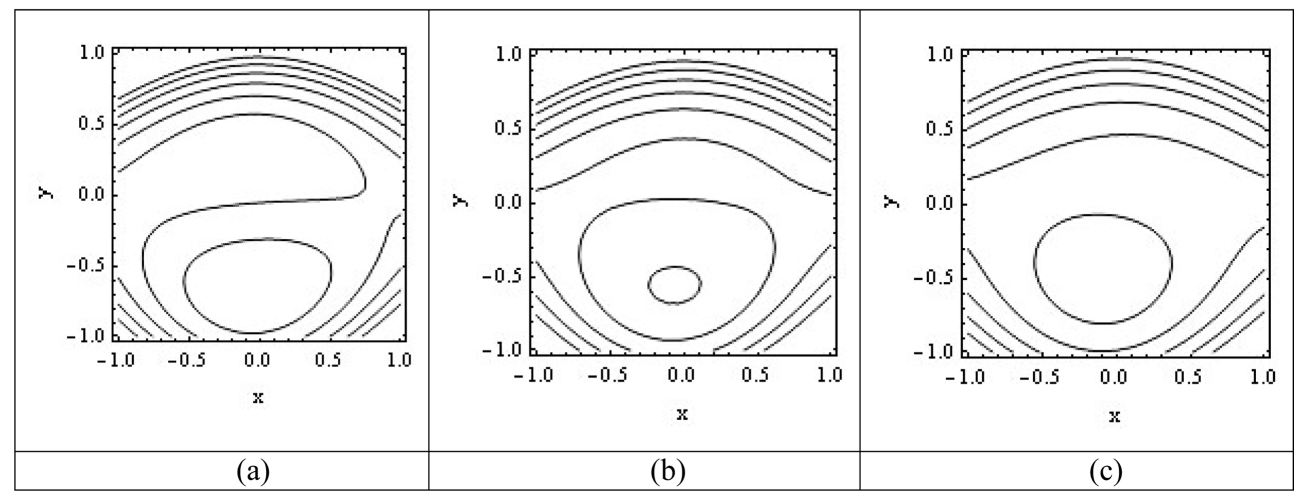

Fig. 14. Streamlines for Hartmann number $M$.

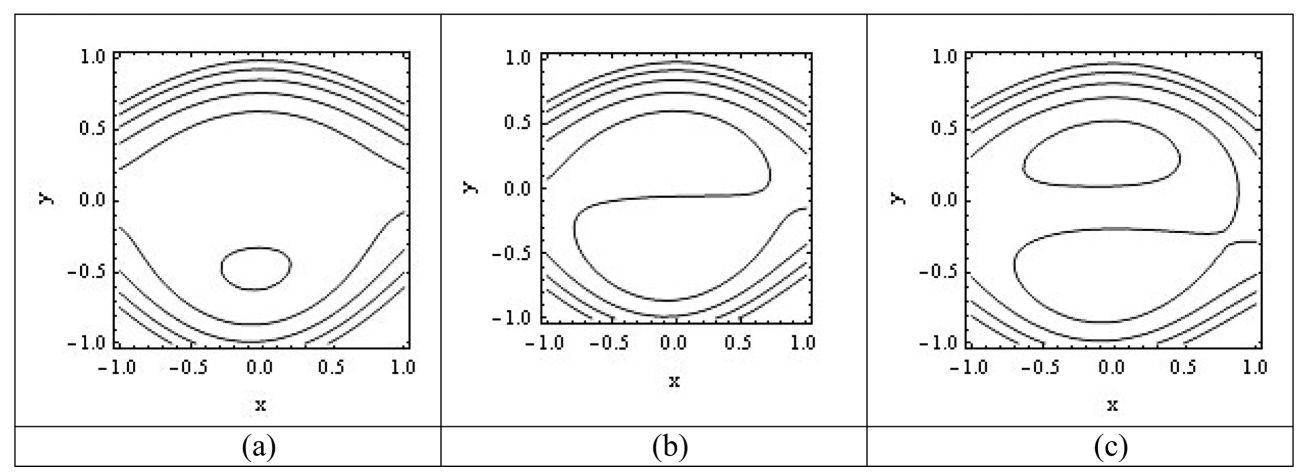

Fig. 15. Streamlines for perturbation parameter $\xi$. 
channel. Figure 12 shows the variation of $\phi$ on the pressure gradient. It is observed that, an increase in $\phi$ decreases the pressure gradient. Figures 13 to 15 portray the effects of trapping phenomenon through stream lines. Figure 13 shows the streamlines for viscosity parameter $\alpha$. It is seen here that as one increase the value of viscosity parameter, the number of bolus is increased. To see the effect of Hartmann number $M$ on the trapping can be depicted in Fig. 14. It is noted that the size of trapped bolus decreases by increasing the values of $\mathrm{M}$. The effect of $\xi$ on the trapping is illustrated in Fig. 15. It is found that the size of the trapped bolus increases with increasing $\xi$ bolus does not occur at $\xi=0$. It is worthwhile mentioning that the streamlines for this particular model are not presented previously. The results obtained in this paper will now be offered experimental authentication.

\section{Conclusion}

In this paper a mathematical model on the peristaltic flow of a Pseudoplastic fluid in an asymmetric channel is investigated. The assumption of low Reynolds number and long wavelength are taken into account. Analysis has been carried under the influence of variable viscosity and magnetic field. Analytical solutions are obtained by regular perturbation method. The main points are as follows:

- The velocity profile increases by increasing the values of $M, \xi$ and $\theta$ at the center of the channel.

- The pressure rise increases with the increase in $M, \xi$ and $\alpha$ in the pumping region.

- The pressure rise decreases by increasing the value of $\phi$ in the pumping region.

- The magnitude of pressure gradient is increasing function of $M, \xi$ and $\alpha$.

- It is worthwhile mentioning that the problem for this particular model is not solved previously even by any traditional perturbation method. However, by taking viscosity parameter $\alpha=0$, the presented result reduces to Newtonian fluid.

\section{Acknowledgment}

R. Ellah grateful to PCST, Ministry of Science and Technology to hone him with the 7th top most Productive Scientist of Pakistan Award in "Category A" for the year 2015.

\section{References}

[1] N. S. Akbar, Zeitschrift für Naturforschung A 70, 745 (2015).

[2] R. Ellahi, A. Riaz, and S. Nadeem, Journal of Mechanics in Medicine and Biology 14, 1450002 (2014).

[3] A Riaz, S. Nadeem, R. Ellahi, and A. Zeeshan, Applied Bionics and Biomechanics 11, 81 (2014).

[4] N. S. Akbar, Applicable Analysis 94, 1420 (2015).

[5] N. S. Akbar, J. Comput. Theor. Nanos. 12, 1546 (2015).

[6] N. S. Akbar, J. Comput. Theor. Nanos. 12, 1553 (2015).

[7] S. Nadeem and Noreen Sher Akbar, Computer Methods in Biomechanics and Biomedical Engineering 14, 987 (2011).

[8] S. Srinivas and M. Kothandapani, International Commun. in Heat and Mass Transfer. 20, 514 (2008).

[9] S. Srinivas and R. Gayathri, Appl. Math. Comput. 215, 185 (2009).

[10] Kh. S. Mekheimer, S. Z. A. Husseny, and Y. Abd Elmaboud, Numer. Methods Partial Differ. Equat. 26, 747 (2010).

[11] V. K. Sud and G. S. Sekhon, Mishra, Bull. Math. Biol. 39, 385 (1977).

[12] A. Yldrm and S. A. Sezer, Mathematical and Computer Modelling 52, 618 (2010).

[13] S. Srinivas and M. Kothandapania, Appl. Math. Comput. 213, 197 (2009).

[14] M. Sheikholeslami, M. G. Bandpy, and H. R. Ashorynejad, Physica A: Statistical Mechanics and its Applications 432, 58 (2015).

[15] A. Ishak, K. Jafar, R. Nazar, and I. Pop, Physica A: Statistical Mechanics and its Applications 388, 3377 (2009).

[16] F. Aman, A. Ishak, and I. Pop, International Communications in Heat and Mass Transfer 47, 68 (2013).

[17] M. I. A. Othman, Mechanics and Mechanical Engineerig 7, 41 (2004)

[18] Y. Abd Elmaboud, Commun. Nonlinear Sci. Num. Sim. 17, 685 (2012).

[19] Kh.S. Mekheimer, Phys. Lett. A 372, 4271 (2008).

[20] S. Akram and S. Nadeem, J. Magn. Magn. Mater. 328, 11 (2013).

[21] Kh. S. Mekheimer and Y. A. Elmaboud, Phys. Lett. A 372, 1657 (2008).

[22] A. El. Hakeem, A. El. Naby, El. A. E. M. Misiery, and I. El. Shamy, Appl. Math. Comput. 158, 497 (2004).

[23] A. El. Hakeem, A. El. Naby, El. A. E. M. Misiery, and I. El. Shamy, J. Phys. A 36, 8535 (2003).

[24] R. Ellahi, Appl. Math. Model 37, 1451 (2013). 